\section{Causes of mutation and Mu excision}

SIR-The results reported by Mittler and Lenski ${ }^{1}$ are completely different from ours ${ }^{2}$. They find that the ara-lac fusion (which allows the Shapiro strain of Escherichia coli to utilize lactose when arabinose is present) occurs at an everincreasing frequency in populations of starving bacteria in liquid medium, reaching a level of more than $10^{-6}$ even in the absence of arabinose and lactose. By contrast, in a large series of experiments conducted over a period of about 6 months, we could not find fusions in liquid, stationary phase cultures until arabinose and lactose were added; in our hands, the frequency of fusion after about a week of incubation at various temperatures was clearly less than $10^{-9}$. Incidentally, the reason we used a rich medium for these experiments was because we did not want there to be any selection pressure for the fusion in the absence of arabinose and lactose (this strain of $E$. coli does not grow well in minimal media and, as Mittler and Lenski observed, tends to die on minimal plates whereas, for some unknown reason, it grows well and does not die once the fusion has occurred).

Several other groups have now done similar experiments. To my knowledge, one laboratory has obtained results like Mittler and Lenski's, one has found what we found and one has reported a mixture. In addition, I have now repeated our experiments using our rich medium and various minimal media, and a detailed description of these experiments was sent to Mittler and Lenski in February. I still fail to detect, in any medium lacking lactose, the presence of any bacteria that are able to form colonies on minimalarabinose-lactose plates as fast as minority populations of cells with fusions that were introduced into these cultures at the start of the experiment.

The basis for this difference in results is not understood. Shapiro showed that the speed of production of fusions in the presence of arabinose and lactose was different for populations derived from sister colonies $^{3}$ and we have found, over the years, that the ability of our standard media to allow certain re-arrangements in $E$. coli seems to be different at different times of year, apparently due to changes in the water supply. Variables like these have not been a significant complication in the study of powerful mutagens, but they give us a tantalizing glimpse of the complexity of what is, for convenience, called "spontaneous" mutation. As Shapiro wrote, "the most pertinent questions in studies of hereditary change must be questions of control and regulation", to which might be added: and the way in which these processes are influenced by circumstances.

The development of livings things has depended on variation plus natural selection. The second of these has received a huge amount of attention since the days of Darwin and Wallace, but the first has hardly been investigated at all. I can think of scarcely a dozen experiments that bear upon the circumstances of what one might call normal spontaneous mutation. And if one is interested in cancer (as I am), then surely one should be asking what are the circumstances determining this lifethreatening form of somatic mutation. JOHN CAIRNS

Department of Cancer Biology,

Harvard School of Public Health,

665 Huntington Avenue,

Boston, Massachusetts 02115, USA

MitTler AND LENSKI REPLY - We stand by our results and interpretations'. After Cairns told us of his difficulty in reproducing the accumulation of excision mutants in minimal-glucose liquid medium (without lactose or arabinose), we repeated the experiment at several different temperatures. In all cases, the frequency of mutants increased to a level significantly greater than that observed in freshly grown cells, although the relative frequency of mutants varies with temperature. J. A. Shapiro (personal communication) has also repeated our experiments, and he too has observed that "continued incubation in minimal-glucose liquid medium results in the accelerated appearance of [excision mutant] colonies on selective agar". As it was Shapiro who originally reported the anomalous behaviour of $E$. coli $\mathrm{MCS} 2$, and who provided that strain to Cairns and to us, we now have even greater confidence in our results and interpretations.

Cairns suggests that the influence of "circumstances", such as "changes in water supply", might somehow account for variation in results. Whether this is so we cannot say. However, the mere existence of variation in the results obtained with MCS2 by different laboratories does not imply that all evidence is equally credible. In particular, we must emphasize that, unlike that of Cairns et al. ${ }^{2}$, our paper' included all the following essential information: (1) explicit laboratory methods; (2) experimental designs with appropriate controls; (3) the level of replication performed for each experiment; (4) the computational method used to estimate mutation rates from directly observable data; and (5) inferential statistics to guide the rejection or acceptance of competing hypotheses. Without an equally thorough accounting of such essential information, one cannot evaluate the cause of any variation between laboratories.

Moreover, we demonstrated' significant effects of several physiological and population-level processes, which Cairns et $a l{ }^{2}$ did not adequately consider. These include: (1) changes in the per capita rate of excision mutation with time, as cells sit starving in the absence of lactose or arabinose; (2) slight growth by MCS2 on lactose and arabinose, or trace contaminants therein; (3) cross-feeding by MCS2 due to metabolites released from $\mathrm{Lac}(\mathrm{Ara})^{+}$excision mutants into the medium; and (4) differences in death rates between MCS2 and $\operatorname{Lac}(\text { Ara })^{+}$excision mutants. The effects of these and other similarly "mundane" processes must be taken into account in further work on the rate of excision of $\mathrm{Mu}$ from MCS2.

Finally, we suggest that future research should consider the role of the $\mathrm{Mu}$ genome ${ }^{t}$. Shapiro (personal communication) has shown that a Mu-encoded function is essential for most of these excisions and we have demonstrated ${ }^{1}$ that the rate of excision increases as the host bacterium starves. A hypothesis consistent with these observations is that the $\mathrm{Mu}$ bacteriophage has evolved (by conventional mutation and natural selection) to use physiological cues indicative of host stress to trigger induction, of which the observed excisions may be a manifestation.

JOHN E. MITTLER RICHARD E. LENSKI

Department of Ecology and

Evolutionary Biology,

University of California,

Irvine, California 92717, USA

1. Mittler, J. E. \& Lenski, R. E. Nature 344, 173-175 (1990).

2. Cairns, J Overbaugh, J. \& Miller, S. Nature 335, 142 145 (1988).

. Shapiro, J. A. Molec gen Genet. 194, 79-90 (1984).

4. Partridge, L. \& Morgan, M. J. Nature 336, 22 (1988)

\section{Radioemission source disputed}

SIR - Maeda and Grebowsky ${ }^{1}$ suggest that impulsive VLF signals observed with the Pioneer Venus Orbiter Electric Field Detector (OEFD) resemble terrestrial VLF saucers. But the similarities between the two phenomena are not as strong as they claim.

First, they use a microdensitometer scan (bandwidth $4 \%$ ) of DE-1 wideband data to show that a narrow-frequency filter will result in impulsive signals. The OEFD bandwidth is $30 \%$. Any impulse caused by a rising or falling tone will therefore last about 7 times longer than shown in their Fig. 2, corresponding to timescales of a few seconds. The bursts observed with the OEFD are known to be shorter than the decay time of the instrument electronics $(\sim 0.7 \mathrm{~s})^{2.3}$

Second, although the free-energy source for VLF saucers has not been observed directly, there is strong evidence that the saucers are generated by return currents associated with the auroral curr- 